OPEN ACCESS

Edited by:

Lu Lu,

Fudan University, China

Reviewed by:

Ji Wang,

Sun Yat-sen University, China Jianqing $\mathrm{Xu}$,

Fudan University, China

*Correspondence:

Linlin Bao

baoll@cnilas.org

Chuan Qin

qinchuan@pumc.edu.cn

tThese authors have contributed equally to this work

Specialty section: This article was submitted to

Virology,

a section of the journal

Frontiers in Microbiology

Received: 18 September 2019

Accepted: 15 October 2019

Published: 30 October 2019

Citation:

Qi F, Liu M, Li F, Lv Q, Wang G, Gong S, Wang S, Xu Y, Bao L and

Qin C (2019) Interleukin-37 Ameliorates Influenza Pneumonia by Attenuating Macrophage Cytokine Production in a MAPK-Dependent

Front. Microbiol. 10:2482. doi: 10.3389/fmicb.2019.02482

\section{Interleukin-37 Ameliorates Influenza Pneumonia by Attenuating Macrophage Cytokine Production in a MAPK-Dependent Manner}

\author{
Feifei Qi',2†, Mingya Liu' ${ }^{1,2 \dagger}$, Fengdi Li ${ }^{1,2}$, Qi Lv ${ }^{1,2}$, Guanpeng Wang ${ }^{1,2}$, Shuran Gong ${ }^{1,2}$, \\ Shunyi Wang ${ }^{1,2}$, Yanfeng $X u^{1}$, Linlin Bao ${ }^{1,2 *}$ and Chuan Qin $^{1,2 *}$ \\ ${ }^{1} \mathrm{NHC}$ Key Laboratory of Human Disease Comparative Medicine, The Institute of Laboratory Animal Sciences, Peking Union \\ Medical College Hospital (CAMS), Beijing, China, ${ }^{2}$ Beijing Key Laboratory for Animal Models of Emerging and Reemerging \\ Infectious, The Institute of Laboratory Animal Sciences, Peking Union Medical College Hospital (CAMS), Beiiing, China
}

Viral pneumonitis caused by influenza A ( $\mathrm{H} 1 \mathrm{~N} 1)$ virus leads to high levels of morbidity and mortality. Given the limited treatment options for severe influenza pneumonia, it is necessary to explore effective amelioration approaches. Interleukin-37 (IL-37) has been reported to inhibit excessive immune responses and protect against a variety of inflammatory diseases. In this study, by using BALB/c mice intranasally infected with A/California/07/2009 (H1N1), we found that IL-37 treatment increases the survival rate and body weight, and reduces the pulmonary index, impaired the lung injury and decreased production of pro-inflammatory cytokines in the BALF and lung tissue. Moreover, IL-37 administration enhanced not only the percentage of macrophages, but also the percentage of IL-18R $\alpha^{+}$macrophages, suggesting that enhancing the macrophages function may improve outcomes in a murine model of $\mathrm{H} 1 \mathrm{~N} 1$ infection. Indeed, macrophages depletion reduced the protective effect of IL-37 during H1N1 infection. Furthermore, IL-37 administration inhibited MAPK signaling in RAW264.7 cells infected with H1N1. This study demonstrates that IL-37 treatment can ameliorate influenza pneumonia by attenuating cytokine production, especially by macrophages. Thus, IL-37 might serve as a promising new target for the treatment of influenza A-induced pneumonia.

Keywords: A/California/07/2009 (H1N1), Interleukin-37, viral pneumonia, macrophages, inflammation

\section{INTRODUCTION}

Influenza H1N1 infection induced "flu"-like illness or pneumonia depends on the infecting strain, host immune system, and environmental factors (Rice et al., 2012; Daoud et al., 2019). H1N1 pneumonia may progress rapidly, resulting in severe respiratory distress syndrome or refractory hypoxemia, is associated with a longer hospital stay with higher mortality compared to bacterial pneumonia (Perez-Padilla et al., 2009; Rello et al., 2009; Hermann et al., 2017). Thus, H1N1 infection is considered a more life-threatening disease (Ramsey and Kumar, 2013; Liu et al., 2016).

Increasing evidence has demonstrated that it is an excessively activated immune response, not a direct viral infection that leads to the increasing influenza pneumonia severity (Morita et al., 2013; Uematsu et al., 2015). Although various prevention and treatment methods have been used for viral diseases, the limited treatment options for severe influenza pneumonia prioritize the need for the discovery of effective therapies. 
Interleukin-37 (IL-37), a novel member of the IL-1 family, inhibits systemic and local inflammation by reducing the levels of pro-inflammatory mediators (Nold et al., 2010; Boraschi et al., 2011; Al-Anazi et al., 2019). IL-37 binds to the IL-18Ra chain, and then recruits TIR-8/IL-1R8/SIGIRR to execute its anti-inflammatory effects (Kumar et al., 2002; Boraschi and Tagliabue, 2013; Dinarello and Bufler, 2013; Lunding et al., 2015; Nold-Petry et al., 2015). IL-37 has been shown to increase the survival rate and body weight, and downregulated the production of IL-6 and IL-17A in a coxsackievirus B3-induced model of murine viral myocarditis (An et al., 2017). In addition, comparing with wild-type (WT) mice, a low dose of mouseadapted H1N1-induced morbidity and the decreases in body weight are significantly attenuated in IL-37tg mice, which express human IL-37 isoform b precursor transgene (Davis et al., 2017). Moreover, IL-37 significantly attenuates pulmonary eosinophilia, CCL11 production and airway hyper-reactivity in a murine asthma model ( $\mathrm{Lv}$ et al., 2018). Increasing evidence suggests that IL-37 can inhibit excessive immune responses and protect against a variety of inflammatory diseases, autoimmune diseases, and tumors. However, little is known about the function of IL-37 in the influenza-infected murine model, particularly the regulatory role in viral pneumonia induced by $\mathrm{A} /$ California/07/2009 (H1N1) infection. Thus, in the present study, we focused on IL-37 treatment in H1N1-infected mice, to investigate the therapeutic effect and the mechanisms by which IL-37 treatment ameliorates influenza pneumonia.

\section{MATERIALS AND METHODS}

\section{Animals and Viruses}

Specific pathogen-free, 4- to 6-week-old female BALB/c mice were obtained from Vital River Laboratories (Beijing, China). The seasonal influenza A virus strain A/California/07/2009 (H1N1) was provided by the Institute of Laboratory Animal Science, Peking Union Medical College, China. All experiments were performed in biosafety level 2 facilities in compliance with governmental and institutional guidelines. The experimental protocol was evaluated and approved by the Institute of Animal Use and Care Committee of the Institute of Laboratory Animal Science, Peking Union Medical College (BLL19004).

\section{Therapeutic Treatments}

Oseltamivir phosphate capsules were purchased from Roche Pharmaceutical Co., Ltd. (Shanghai, China). The pGEM-T-IL-37b plasmid was kindly supplied by Dr. RF. Wei., Institute of Laboratory Animal Science, Peking Union Medical College, China. Individual mice were anesthetized with tribromoethanol and inoculated intranasally with $50 \mu \mathrm{l}\left(10^{4.3} \mathrm{TCID}_{50}\right)$ of allantoic fluid containing influenza A/California/07/2009 (H1N1) virus. Subsequently, the mice were chronically intragastrically administered oseltamivir phosphate $(30 \mathrm{mg} / \mathrm{kg})$ for 5 days, and the animals were inoculated with IL-37 $(12.5 \mu \mathrm{g} / \mathrm{kg})$ via intravenous or intranasal administration at three separate time points $(2,24$, and $48 \mathrm{~h}$ post infection). Seven mice were selected randomly from each group for monitoring the disease signs, weight loss, and mortality daily up to 14 days post inoculation (d.p.i.). The remaining mice in each group were euthanized at 6 d.p.i. and blood samples, bronchoalveolar lavage fluid (BALF), and lung tissues were collected for the assessment of lung histology, pro-inflammatory cytokines, and immune cell counts.

\section{Preparation of Single Cell Suspensions From the Lung}

Mice were anesthetized and the lung was flushed in situ with $20 \mathrm{ml}$ of phosphate-buffered saline (PBS) via cannulation of the heart to remove the intravascular blood pool. Minced lung tissues were incubated at $37^{\circ} \mathrm{C}$ for $1 \mathrm{~h}$ on a rocker with $200 \mu \mathrm{g} /$ $\mathrm{ml}$ collagenase D and $40 \mu \mathrm{g} / \mathrm{ml}$ DNase I (Roche Molecular Biochemicals) in $10 \mathrm{ml}$ of DMEM supplemented with $10 \%$ FBS. Single cell suspensions from the digested lung were filtered through a $75-\mu \mathrm{m}$ strainer and then collected through densitygradient centrifugation with lymphocyte separation solution. The immune cells were washed twice with Hank's solution and suspended in Hank's solution.

\section{Flow Cytometry Analysis}

Cells were pre-incubated with Zombie Aqua $^{\mathrm{TM}}$ Fixable Viability Kit (Biolegend) and purified rat anti-mouse CD16/CD32 (Mouse BD Fc Block ${ }^{\mathrm{TM}}, 2.4 \mathrm{G} 2$, BD Pharmingen ${ }^{\mathrm{TM}}$ ) ice for $15 \mathrm{~min}$ at room temperature. For the extracellular cell marker analysis, the cells were incubated with the following fluorescein-conjugated antibodies for $30 \mathrm{~min}$ : BV421-anti-CD11b (M1/70, Biolegend), FITC-anti-CD45 (30-F11, Biolegend), BV605-anti-F4/80 (BM8, Biolegend), PE-anti-IL-18Ra (Miltenyi Biotec), AF647-antiSIGIRR (Santa Cruz), BV421-anti-CD3e (145-2C11, Biolegend), PE-Cy7-anti-CD4 (RM4-5, BD Biosciences), and PerCP-Cy5.5anti-CD8a (53-6.7, BD Biosciences). Finally, samples were acquired using a fluorescence-activated cell sorting (FACS) Aria II system (BD Biosciences). The data were analyzed using a Kaluza analysis and FlowJo 10.1 software.

\section{Preparation of Lung Homogenate Supernatant}

Lung homogenates were prepared by homogenizing perfused whole lung tissue using an electric homogenizer for $2 \mathrm{~min}$ $30 \mathrm{~s}$ in $1 \mathrm{ml}$ of PBS. The homogenates were centrifuged at $3,000 \mathrm{rpm}$ for $10 \mathrm{~min}$ at $4^{\circ} \mathrm{C}$. The supernatant was collected and stored at $-80^{\circ} \mathrm{C}$.

\section{Analysis of Bronchoalveolar Lavage Fluid}

Bronchoalveolar lavage fluid (BALF) was collected by washing the lungs of sacrificed mice twice with $1 \mathrm{ml}$ PBS. The PBS was then recovered after $1 \mathrm{~min}$ and centrifuged at $1,500 \mathrm{rpm}$ for $10 \mathrm{~min}$ at $4^{\circ} \mathrm{C}$. The supernatant was collected and stored at $-80^{\circ} \mathrm{C}$. Total cellular infiltration in the BALF was assessed using a hemocytometer; cytosine slides were fixed and stained with WrightGiemsa stain, and the composition was assessed in a blinded manner by counting 200 or more cells using a light microscope.

\section{Clodronate Treatment}

To deplete macrophages, ready-made clodronate liposomes and control liposomes (FormuMax; CA, USA) were intranasally 
administered to mice using the manufacturer's recommending dose 1 day before and 1 day after A/California/07/2009 (H1N1) infection. Mice were monitored for signs of disease, weight loss, and mortality upto 14 d.p.i.

\section{Quantification of Cytokines}

The concentrations of granulocyte colony-stimulating factor (G-CSF), granulocyte-macrophage colony-stimulating factor (GM-CSF), interferon- $\gamma$ (IFN- $\gamma)$, monokine induced by IFN- $\gamma$ (MIG), interleukin (IL)-1 $\alpha$, IL-1 $\beta$, IL-4, IL-5, IL-6, IL-10, IL-12/IL-23p40, IL-13, IL-17A, RANTES, monocyte chemoattractant protein 1 (MCP-1), macrophage inflammatory protein $1 \alpha$ (MIP-1 $\alpha)$, MIP-1 $\beta$, and tumor necrosis factor (TNF) in the serum and BALF samples were determined by flow cytometry (FACS Aria II, BD, USA) using the Cytometric Beads Array (CBA) Kits, according to the manufacturers' instructions (BD, USA). Briefly, $50 \mu \mathrm{l}$ of each testing sample were labeled in duplicate with equal volumes of diluted FlexSet capture beads at room temperature for $1 \mathrm{~h}$ and treated with PE-conjugated detection reagent. After washing, the captured cytokines and chemokines were analyzed by flow cytometry.

\section{Hematoxylin and Eosin Staining}

For each mouse, the whole right lung was fixed in $10 \%$ formalin for $24 \mathrm{~h}$ and then embedded in paraffin for histological examination. The lung tissue sections $(4 \mu \mathrm{m})$ were deparaffinized and hydrated using xylene and an alcohol gradient and then, stained with Hematoxylin and Eosin (H\&E). The histopathology of the lung tissue was observed by light microscopy.

\section{Quantitative Real-Time Polymerase Chain Reaction Analysis}

Total RNA was isolated from individual samples using an RNeasy Mini kit, according to the manufacturer's instructions (Qiagen, Hilden, Germany). The RNA was reversely transcribed into cDNA using random primers and a SuperScript II reverse transcriptase reaction mixture (Invitrogen). The target gene mRNA transcripts were determined by RT-PCR using SYBR Green PCR Master Mix, specific primers, and a 7500 PCR system (ABI, USA). Primer sets for individual genes are shown in Table $\mathbf{1}$.

TABLE 1 | Primer sequences used for RT-PCR analysis.

\begin{tabular}{|c|c|c|}
\hline Gene & Forward $\left(5^{\prime}-3^{\prime}\right)$ & Reverse $\left(5^{\prime}-3^{\prime}\right)$ \\
\hline$\beta$-actin & CAACGAGCGGTTCCGATG & GCCACAGGATTCCATACCCA \\
\hline IL-6 & TCTATACCACTTCACAAGTCGGA & GAATTGCCATTGCACAACTCTIT \\
\hline $\mathrm{IL} 1 \alpha$ & TCTCAGATTCACAACTGTTCGTG & AGAAAATGAGGTCGGTCTCACTA \\
\hline $\mathrm{IL}-1 \beta$ & CAACCAACAAGTGATATTCTCCATG & GATCCACACTCTCCAGCTGCA \\
\hline RANTES & GCTGCTाTGCCTACСTCTCC & TCGAGTGACAAACACGACTGC \\
\hline IP10 & CCAAGTGCTGCCGTCATITC & GGCTCGCAGGGATGATTTCAA \\
\hline MCP1 & AGTAGGCTGGAGAGCTACAA & GTATGTCTGGACCCATTCCTTC \\
\hline MIP1 $\alpha$ & TTCTCTGTACCATGACACTCTGC & CGTGGAATCTTCCGGCTGTAG \\
\hline MIP1 $\beta$ & TTCCTGCTGTTCTCTTACACCT & СTGTCTGCCTCTITGGTCAG \\
\hline MIG & TCCTITGGGCATCATCTTCC & TाTGTAGTGGATCGTGCCTCG \\
\hline $\mathrm{IFN}-\gamma$ & TATCTGGAGGAACTGGCAAA & GGTGTGATTCAATGACGCTT \\
\hline IL $18 R \alpha$ & TCACCGATCACAAATTCATGTGG & TGGTGGCTGTTTCATTCCTGT \\
\hline
\end{tabular}

The results are normalized to $\beta$-actin expression and presented as the fold change in mRNA expression (fold change $=2^{-\triangle \triangle C T}$ ).

\section{Cells}

Murine macrophage cell lines (RAW264.7) were maintained in Dulbecco's modified Eagle's medium (Gibco, Life Technologies, New York) supplemented with 10\% FBS, $100 \mathrm{IU} / \mathrm{ml}$ penicillin, and $100 \mu \mathrm{g} / \mathrm{ml}$ streptomycin and were incubated at $37^{\circ} \mathrm{C}$ with $5 \% \mathrm{CO}_{2}$. RAW264.7 cells in six-well plates were infected with $\mathrm{H} 1 \mathrm{~N} 1$ at a multiplicity of infection (MOI) of 0.01 for $1 \mathrm{~h}$ absorption at $37^{\circ} \mathrm{C}$. Then, the cells were washed and cultured with $2 \mathrm{ml}$ of serum-free DMEM containing TPCK-treated trypsin $(0.5 \mathrm{mg} / \mathrm{ml})$ antibiotics and $70 \mu \mathrm{M}$ oseltamivir phosphate with or without IL-37b for $72 \mathrm{~h}$. The cells were collected at 0,12 , and $24 \mathrm{~h}$ post infection to detect the expression of cytokines such as IL- 6, MCP-1, TNF- $\alpha$, IL- $1 \beta$, and IL- $1 \alpha$. The protein levels of MAPKs and NLRP3 were detected by western blot assay at 0,30 , and $60 \mathrm{~min}$ after infection.

\section{Western Blot Assay}

The expression of GAPDH, p38, phospho-p38 (p-p38), ERK, phospho-ERK (p-ERK), NF- $\kappa B$, JNK, phospho-JNK (p-JNK), and NLRP3 (Cell Signaling, Boston, MA, USA) was assessed by western blotting. Protein bands were detected with a chemiluminescent imaging system (Amersham, Freiburg, Germany). The expression of a target protein was normalized to that of GAPDH.

\section{Statistical Analysis}

The data are presented as the mean \pm SEM. Analysis of variance (ANOVA) was used to analyze the differences between three or more groups, and $t$ tests were used to analyze the differences between two groups. Statistical graphs were obtained using GraphPad Prism 5 software. Differences were considered statistically significant at values of $p<0.05$.

\section{RESULTS}

\section{Interleukin-37 Treatment Reduces the Body Weight Recovery Time and Improves the Survival Rate in H1N1-Infected Mice}

To explore the efficacy of the recombinant IL-37 protein, BALB/c mice challenged intranasally with $50 \mu \mathrm{H} 1 \mathrm{~N} 1$ were treated with oseltamivir phosphate for 5 days with/without recombinant IL-37 for 7 days at 2 h.p.i. As shown in Figure 1A, the body weights of mice treated for 7 days (oseltamivir+IL-37 7d) were not obviously different from those of mice in the oseltamivir phosphate group. Nevertheless, in this study, we extended the IL-37 administration time to 9 days (oseltamivir+IL-37 9d), and the death and body weight changes in the mice $(n=7)$ were monitored for 14 d.p.i. The results showed that the body weights of mice began to increase from 7 d.p.i., which was 2 days earlier than the body weights of mice in the oseltamivir phosphate group began to increase (Figure 1A). A total of $71 \%$ of the mice from the oseltamivir+IL-37 9d group survived, whereas the survival rate in the oseltamivir phosphate group was $57 \%$ survival. All of the mice in the model group died (Figure 1B), suggesting that IL-37 treatment for 9 days advanced the onset of body weight recovery and improves the survival rate in $\mathrm{H} 1 \mathrm{~N} 1$-infected mice. 

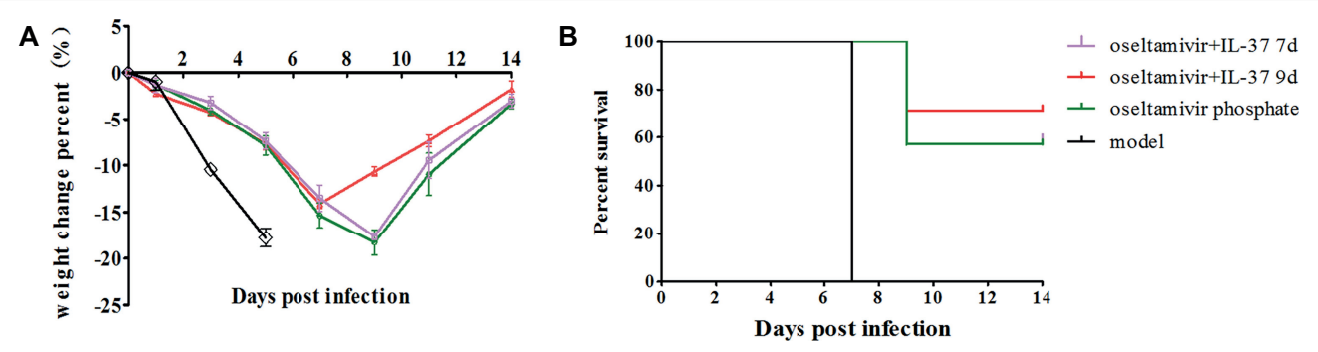

FIGURE 1 | IL-37 treatment reduces the time to body weight recovery onset and decreases mortality in mice during influenza infection. Mice were infected intranasally with $10^{4.6} \mathrm{TCID}_{50}$ of influenza A ( $\left.\mathrm{H} 1 \mathrm{~N} 1\right)$ virus; body weight $\mathbf{( A )}$ and mortality rates (B) were recorded at two different time points of intravenous IL-37 administration. Data are presented as the average values from three independent experiments $\pm \operatorname{SD}(n=7$ per group).

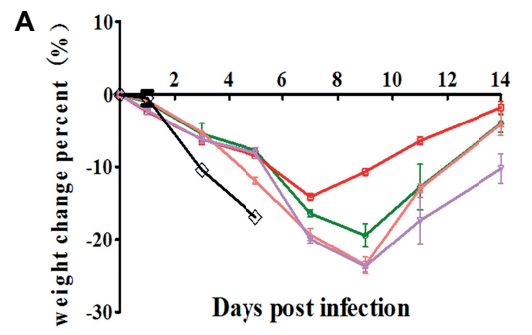

C

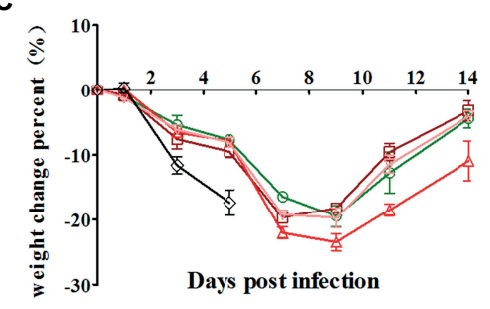

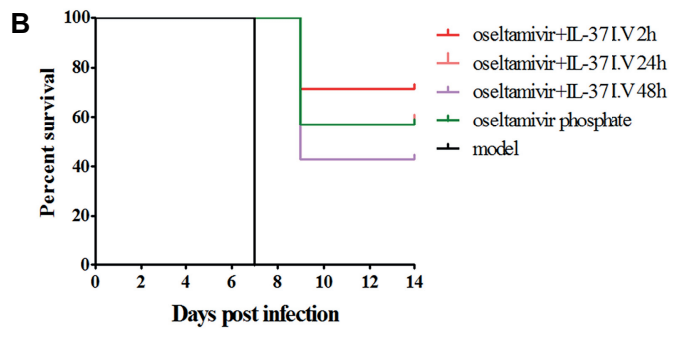

D

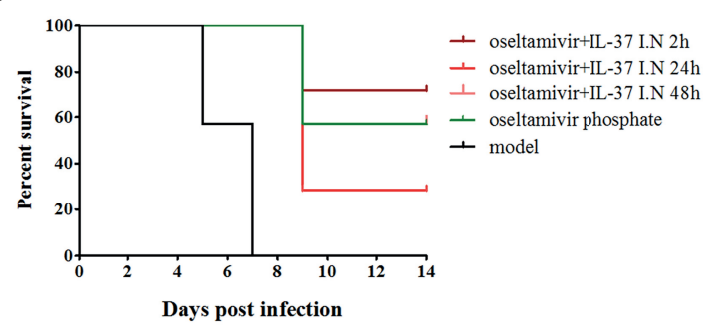

FIGURE 2 | IL-37 intravenous administration offers enhanced protection against influenza challenge in mice. BALB/c mice ( $n=7$ in each group) treated with or without IL-37 were divided into eight groups: the H1N1-infected group (model) and oseltamivir phosphate, intravenous oseltamivir phosphate combined with IL-37 at three separate time points (oseltamivir+IL-37 I. V $2 \mathrm{~h}$, oseltamivir+IL-37 I. V $24 \mathrm{~h}$, oseltamivir+IL-37 I. V $48 \mathrm{~h}$ ) or intranasal oseltamivir phosphate combined with IL-37 at three separate time points (oseltamivir+IL-37 I. N 2 h, oseltamivir+IL-37 I. N 24 h, oseltamivir+IL-37 I. N 48 h) treatment groups. The body weights (A,C) and mortality rates (B,D) of mice treated via intravenous or intranasal routes were monitored. Data are presented as the average values from two independent experiments $\pm \mathrm{SD}(n=7$ per group).

\section{Intravenous Interleukin-37 Administration in Mice Enhances the Protection Against Influenza Challenge}

To further explore the best time point and route of administration for IL-37, after infection, the BALB/c mice were divided into eight groups: $\mathrm{H} 1 \mathrm{~N} 1$ infection (model), oseltamivir phosphate, oseltamivir phosphate combined with intravenous IL-37 administration at three separate time points (oseltamivir+IL-37 I. V $2 \mathrm{~h}$, oseltamivir+IL-37 I. V $24 \mathrm{~h}$, and oseltamivir+IL-37 I. V $48 \mathrm{~h}$ ) or oseltamivir phosphate combined with intranasal IL-37 administration at three separate time points (oseltamivir+IL-37 I. N 2 h, oseltamivir+IL-37 I. N 24 h, and oseltamivir+IL-37 I. N 48 h). A model group was also administered PBS. Seven mice were selected randomly from each group, and mortality rates and body weight changes in the mice were monitored for 14 d.p.i. Treatment with IL-37 protein in combination with oseltamivir phosphate reduced the time to the onset of body weight recovery, and this reduction varied based on the different administration times. As shown in Figure $\mathbf{2 A}$, the body weights of mice infected with $\mathrm{H} 1 \mathrm{~N} 1$ decreased and began to increase from the 7th day of IL-37 plus oseltamivir phosphate treatment at 2 h.p.i. (oseltamivir+IL-37 I.V $2 \mathrm{~h}$ ), whereas the body weights of mice in the oseltamivir phosphate group began to increase on the 9th day; in the model group, the body weight continued to decrease until death. Moreover, animals that received PBS succumbed to infection 5-7 days after viral challenge. When therapeutic treatment was started at 24 h.p.i, $57 \%$ of the infected mice survived, which was the same rate as the mice treated with oseltamivir phosphate only. The administration of IL-37 at 2 h.p.i. increased the mouse survival rate to $71 \%$. However, in the group administered IL-37 at 48 h.p.i, the mortality rate increased (Figure 2B). 
Similar to the results shown in Figure 2, the survival rate of mice intranasally administered IL-37 at 2 h.p.i., was significantly enhanced compared to that of mice in the oseltamivir phosphate group (Figure 2D). However, the body weight in the intranasal IL-37 treatment group was not significantly increased compared with that in the oseltamivir phosphate group; however, the body weight decrease time was shortened and remained stable at 7-9 d.p.i (Figure 2C). These results demonstrate that intravenous IL-37 administration at 2 h.p.i significantly decreases the mortality of mice infected with $\mathrm{H} 1 \mathrm{~N} 1$ and shortens the recovery time of infected mice, so intravenous IL-37 administration at 2 h.p.i offers enhanced protection against influenza challenge in mice.

\section{Interleukin-37 Treatment Reduces Lung Damage in Mice Infected With H1N1 Virus}

In addition, to further validate the therapeutic effect of IL-37, lung tissue was collected to monitor the pulmonary indexes and lung histology. As expected, the IL-37 combined with oseltamivir phosphate administration group had significantly lower pulmonary index values than the other groups (Figure 3A). The BAL fluid was gathered 6 days after infection or IL-37 treatment. The total cell number in the BALF was increased significantly after infection (Figure 3B). However, IL-37 treatment evidently diminished $\mathrm{H} 1 \mathrm{~N} 1$-induced neutrophilic and eosinophilic airway inflammation (Figure 3C). Additionally, $\mathrm{H} \& \mathrm{E}$ staining of the lung tissue samples showed that the lungs in the model group exhibited many merged, inflated, or enlarged alveoli as well as an increase in the exudation of inflammatory proteins in the alveolar spaces at 6 d.p.i., which was largely decreased in the IL-37-treated group (Figure 3D). These results further indicate that IL-37 could be a useful therapeutic agent in mice with $\mathrm{H} 1 \mathrm{~N} 1$ infection.

\section{Interleukin-37 Inhibits the Production of Inflammatory Cytokines in H1N1-Infected Mice}

IL-37, as a potent inhibitor of innate immunity, can shift the cytokine equilibrium away from excessive inflammation (Teng et al., 2014). Thus, to more accurately assess the efficacy
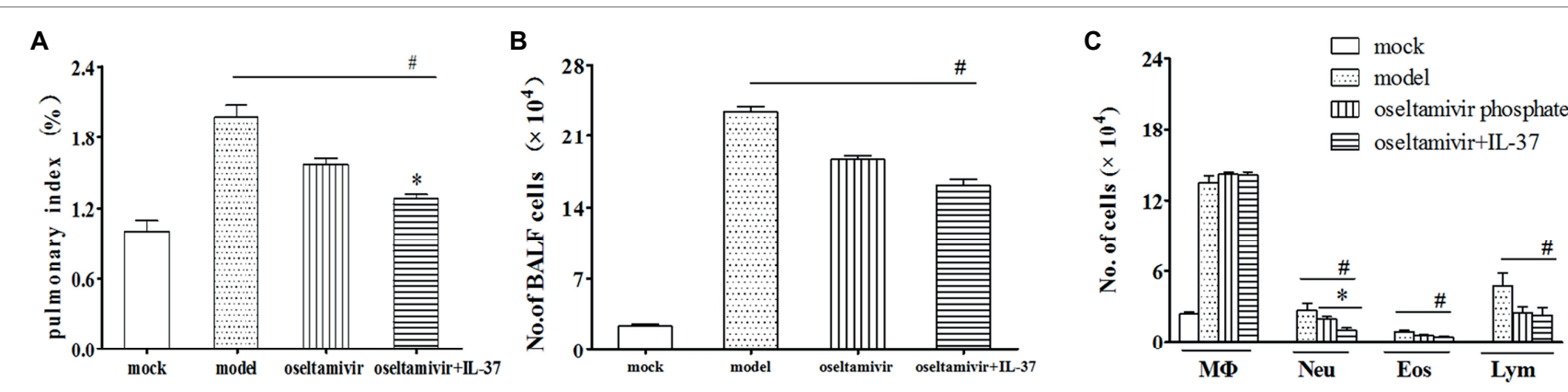

D
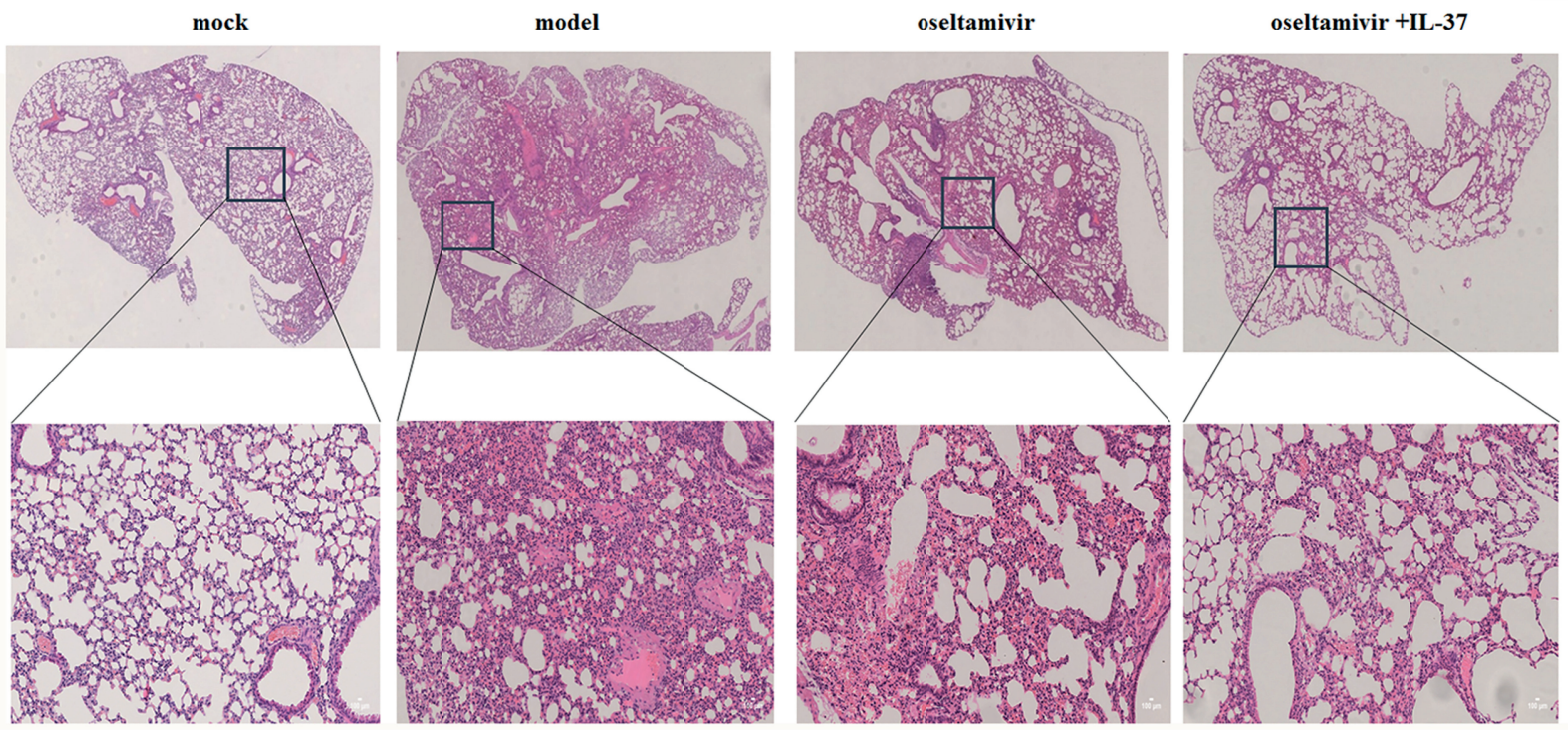

FIGURE 3 | IL-37 treatment attenuates H1N1-induced lung tissue damage in vivo. Lungs were obtained from different groups of mice, and the pulmonary index on day 6 post infection was monitored (A). The BALF was harvested on day 6 d.p.i, the number of total BALF cells (B) and the proportions of different leukocyte subtypes in the BALF (C) were calculated. (D) Mouse lung tissues were stained with H\&E. Data are representative of three independent experiments with three mice per group (100x magnification). Data are representative of three independent experiments with three mice for each group. * ${ }^{*}$ ignificant difference $(p<0.05)$, compared with oseltamivir-inoculated mice. "Significant difference $(p<0.05)$, compared with H1N1-infected mice. 
of IL-37 during H1N1 infection, the mRNA expression and the protein production levels of IL-6, TNF- $\alpha$, MCP-1, IL- $1 \alpha$, IL- $1 \beta$, MIP- $1 \alpha$, MIP- $1 \beta$, IP-10, MIG, RANTES, IFN- $\gamma$ and IL-10 were detected by RT-PCR and CBA in the lung tissue, BALF and serum samples on day 6 after H1N1 infection. As expected, IL-37 treatment inhibited the increase in levels of MCP-1, IL- $1 \beta$, MIP- $1 \alpha$, MIP- $1 \beta$, MIG, IFN- $\gamma$ and RANTES in the lungs of the model group (Figure $4 \mathbf{A}$ ). Paralleling the decreased production of cytokines, the upregulation of MCP-1, IL-1 $\beta$, IL-6, IP-10, MIG, and RANTES mRNA expression was markedly reduced in the IL-37 treatment group (Figure 4D). In addition, it is worth mentioning that the expression of MCP- 1 and IL- $1 \beta$, especially the level of MCP- 1 in the oseltamivir group, was higher than that in the model group; however, oseltamivir plus IL-37 treatment corrected the increase in MCP-1 expression (Figure 4D). Moreover, the mRNA expression of the anti-inflammatory cytokine IL-10 was downregulated in the IL-37-treated group (Figure 4D); however, the production of IL-10 protein in the lungs was not significantly changed (data not shown).

Furthermore, compared with that in the model and oseltamivir groups, the upregulated production of MCP-1, IL-6 and IFN- $\gamma$ in the BALF (Figure 4B) and serum (Figure 4C) was markedly reduced in the IL-37 treatment group. These results indicate that IL-37 exerts a protective effect by regulating the levels of inflammatory cytokines, particularly by regulating macrophage cytokine production.

\section{Macrophage Percentages Are Increased in Interleukin-37 Treated Mice}

To evaluate the roles of immune cells in the IL-37-mediated protection against influenza A (H1N1) infection, the changes in the numbers of macrophages and $\mathrm{T}$ cells in the lungs of different groups at multiple time points were analyzed using flow cytometry. In fact, the percentages of $\mathrm{CD} 4^{+}$and $\mathrm{CD} 8^{+}$, IL- $18 \mathrm{Ra}^{+} \mathrm{CD}^{+}$or $\mathrm{CD}^{+} \mathrm{T}$ cells in the IL-37 treatment group were not obviously different compared with those in the model group (Supplementary Figure S1). However, an increase in the percentage of macrophages, which were identified as $\mathrm{CD} 45^{+} \mathrm{F} 4 / 80^{+} \mathrm{CD} 11 \mathrm{~b}^{+}$cells, was observed in the lungs of mice in the IL-37 administration group, peaking at day 6 post infection (Figure 5A). Paralleling the augmented macrophage percentage, the percentage of IL-18R ${ }^{+}$macrophages was markedly enhanced in the lungs of IL-37-treated mice (Figure 5B). These results show that IL-37 administration impairs the decrease in the macrophage population in the lungs, indicating that macrophages may exert immunoprotective effects in mice treated with IL-37 during H1N1 infection.

\section{Depleting Macrophages Reduces the Protective Effect of Interleukin-37 During Influenza Virus Infection}

As macrophages may exert immunoprotective effects in H1N1infected mice treated with IL-37, we intranasally administered clodronate liposomes, which have been shown to deplete macrophages in the lungs (Tate et al., 2010; Wong and Smith, 2017), to mice during influenza virus infection to test the dependency of the IL-37 treatment-induced reduction in mortality on macrophages. As expected, under the same conditions, the clodronate liposome administration group reached its lowest weight at 9 d.p.i. and the body weight change did not recover in the control liposome group (Figure 6A). Moreover, clodronate liposome treatment significantly increased the mortality rate (Figure 6B). These data further demonstrate that macrophages are critical for the protective effect of IL-37 during influenza virus infection.

\section{Interleukin-37 Inhibits the Expression of Inflammatory Cytokines in a MAPK- Dependent Manner in vitro}

To clearly demonstrate the anti-inflammatory efficacy of IL-37, following H1N1 infection, murine macrophage RAW264.7 cells were treated with IL-37 for different times (12 and $24 \mathrm{~h})$. The levels of inflammatory factors in the cells were determined by real-time PCR. As shown in Figure 7A, IL-6 mRNA expression at all time points was obviously downregulated in the IL-37 treatment groups compared with the oseltamivir group. Similar inhibitory effects were also observed on TNF- $\alpha$, IL-1 $\beta$ and MIP-1 $\beta$ expression (Figure 7A).

To further explore the underlying mechanisms of IL-37, we examined the expression of PRR-related protein phosphorylation. For this purpose, western blot analyses were performed using the cell lysates of RAW264.7 cells treated with or without IL-37 to analyze the phosphorylation of MAPKs and GAPDH. In addition, NLRP3 protein production was detected. As shown in Figure $\mathbf{7 B}$, compared with that in the infection and oseltamivir groups, the phosphorylation of ERK1/2 and p38 MAPK was significantly reduced in the IL-37 treated RAW264.7 cell group. Similarly, the ratio of NLRP3 in the IL-37-treated group was significantly decreased in vitro. These results show that IL-37 treatment inhibits the production of macrophage inflammatory cytokines induced by $\mathrm{H} 1 \mathrm{~N} 1$ infection in a MAPK-dependent manner.

\section{DISCUSSION}

Influenza viruses cause seasonal epidemics and sporadic pandemics, and are a major burden on human health. The rapid development of viral pneumonitis induced by aggressive inflammation resulting in high morbidity and mortality, emphasizing the importance of exploring effective approaches to ameliorate the viral pneumonia during $\mathrm{H} 1 \mathrm{~N} 1$ infection (Morita et al., 2013). IL-37 has been shown to block the deleterious effects of pro-inflammatory stimuli or conditions in numerous models (Barbier et al., 2019; Theoharides et al., 2019), it is essential for the inhibition of innate immunity and inflammation and plays a role in the inhibition of cytokine and chemokine production, and inflammatory cell infiltration (Theoharides et al., 2019). Increasing evidence suggests that the anti-inflammatory cytokine IL-37, improve functional outcomes in combination, including neuroprotection and reduced of lung infection burden (Zhang et al., 2019). 

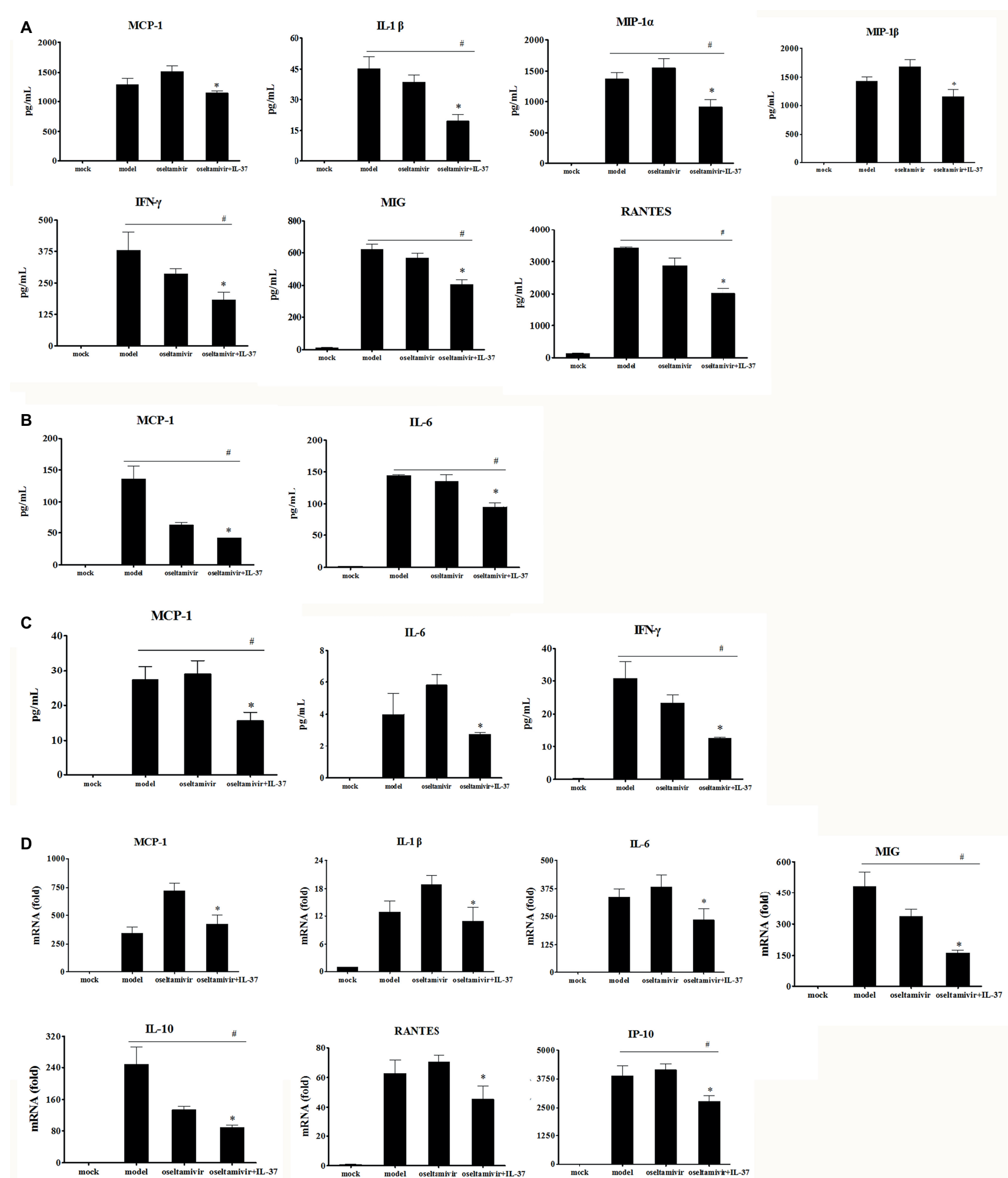

FIGURE 4 | IL-37 treatment modulates immune responses to H1N1 infection. BALF, serum and lung homogenates were harvested 6 d.p.i. following IL-37 treatment. The levels of G-CSF, GM-CSF, IFN- $\gamma$, MIG, IL-1 $\alpha$, IL-1 $\beta, I L-4, I L-5, I L-6, I L-10, I L-12 p 70, I L-13, I L-17 A$, RANTES, MCP-1, MIP-1 $\alpha$, MIP-1 $\beta$, and TNF, in lung homogenates (A), BAL fluid (B) as well as serum samples (C) were measured quantitatively using a Cytometric Beads Array. There were no significant differences in the concentrations of other cytokines and chemokines tested (data not shown). (D) The relative mRNA expression of cytokines in lung tissues were detected by real-time RT-PCR. Data are representative of three independent experiments with three mice for each group. ${ }^{\star}$ Significant difference $(p<0.05)$, compared with oseltamivir-inoculated mice. * Significant difference $(p<0.05)$, compared with H1N1-infected mice. 


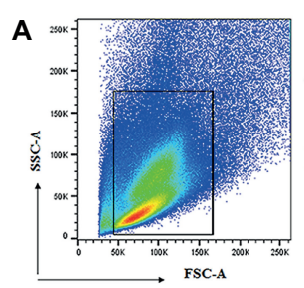

mock
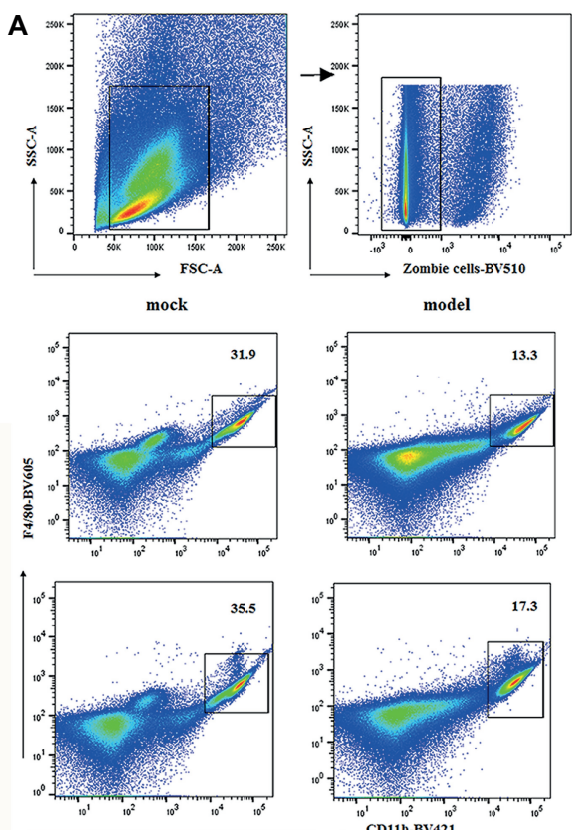

model
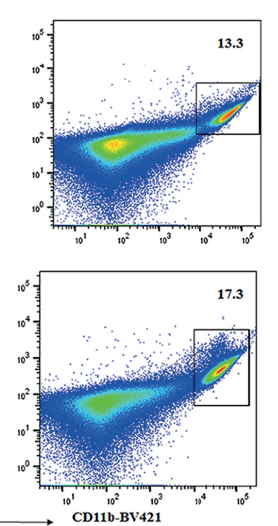

B
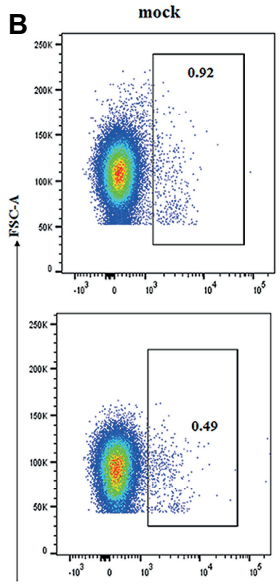
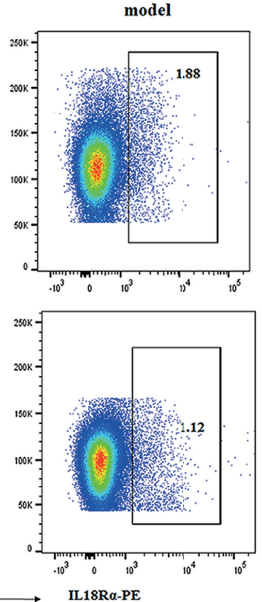

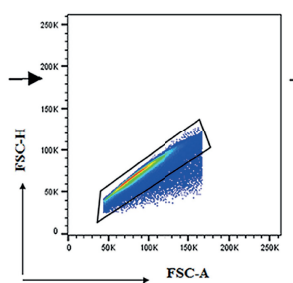

$\longrightarrow$ FSC-A
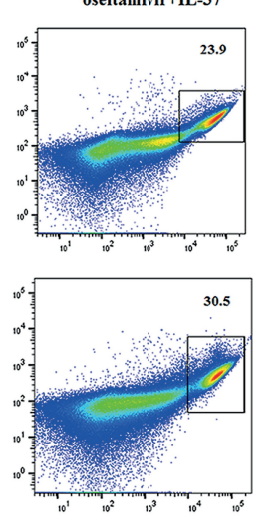

oseltamivir+IL-37
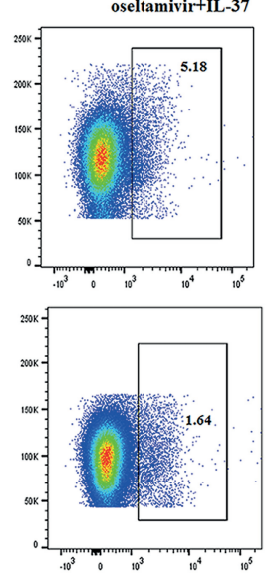
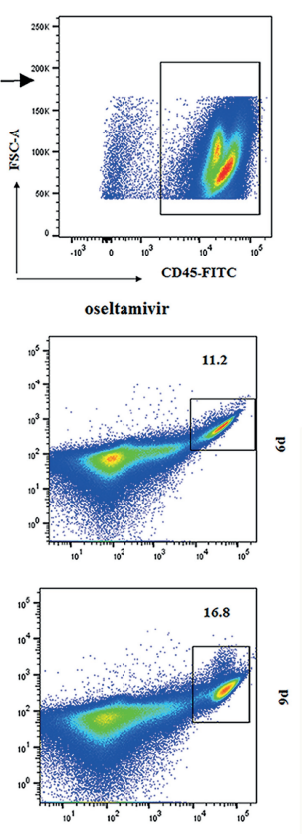

$\square$ mock

西 oseltamivir

○ oseltamivir+IL-37
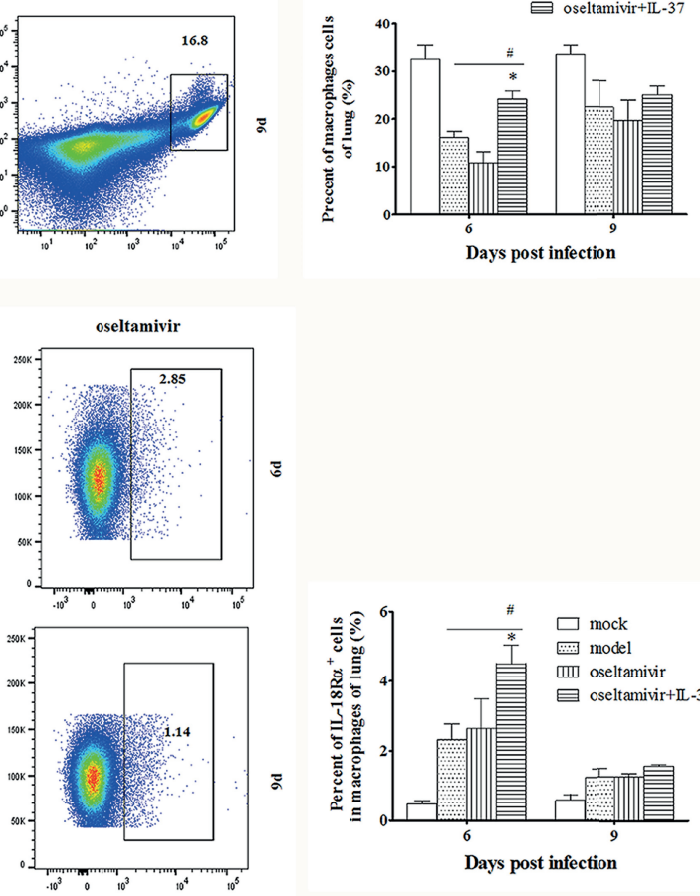

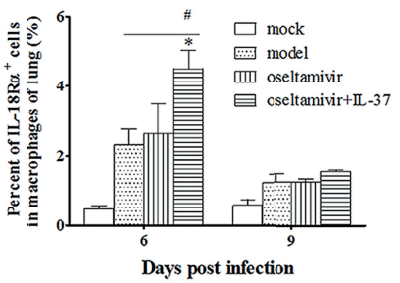

FIGURE 5 | The macrophage percentages are increased in IL-37-treated mice. The percentage of CD45+F4/80+CD11 b macrophages (A) and IL-18R $\alpha^{+}$macrophages (B) in the lungs of mice was determined by flow cytometry at the indicated time points. Data are representative of three independent experiments with three mice for each group. *Significant difference $(p<0.05)$, compared with oseltamivir-treated mice. "Significant difference $(p<0.05)$, compared with H1N1-infected mice.
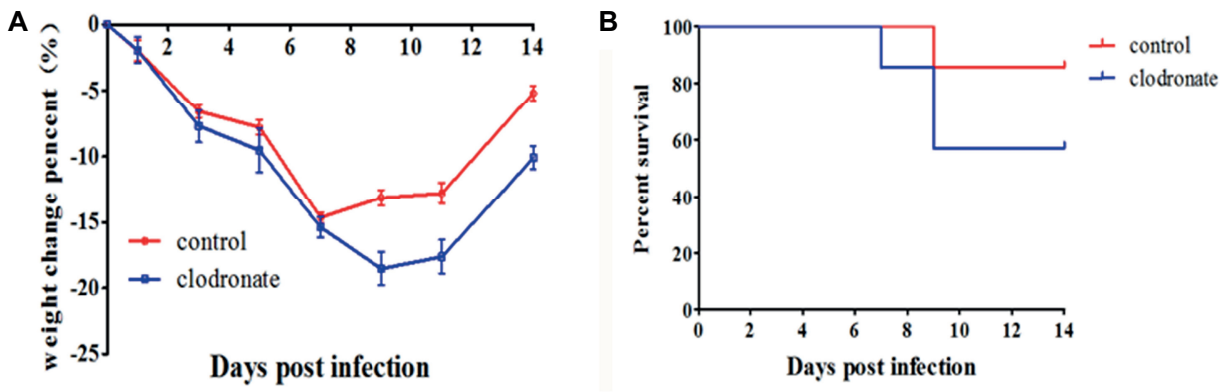

FIGURE 6 | Macrophage depletion reduces the protective effect of IL-37 during influenza virus infection. Body weights (A) and survival rates of (B) mice treated with clodronate liposomes or control liposomes during IL-37 administration. Data are presented as the average values from two independent experiments \pm SD $(n=7$ per group). 
A

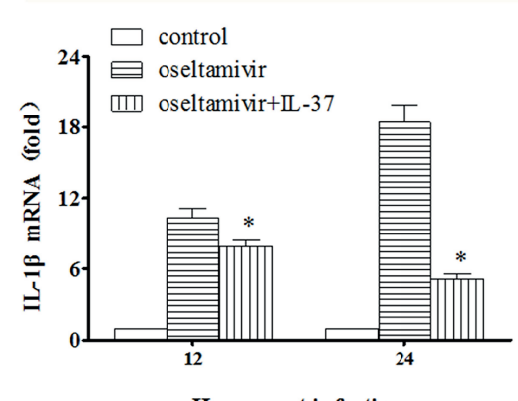

Hours post infection

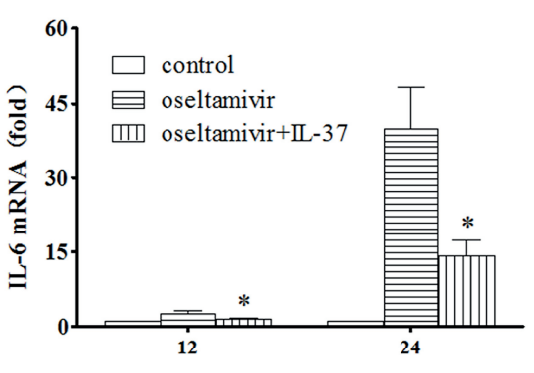

Hours post infection

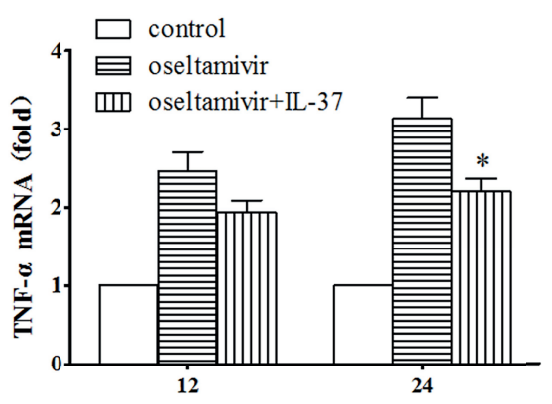

Hours post infection

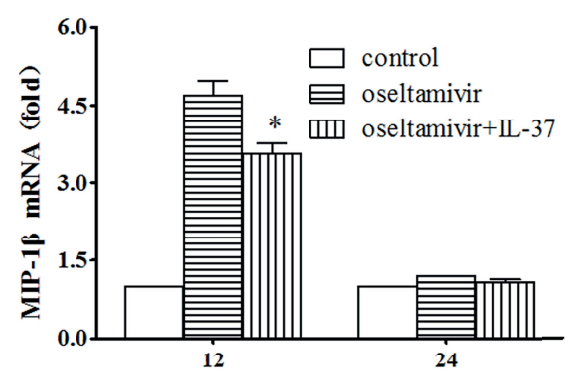

Hours post infection
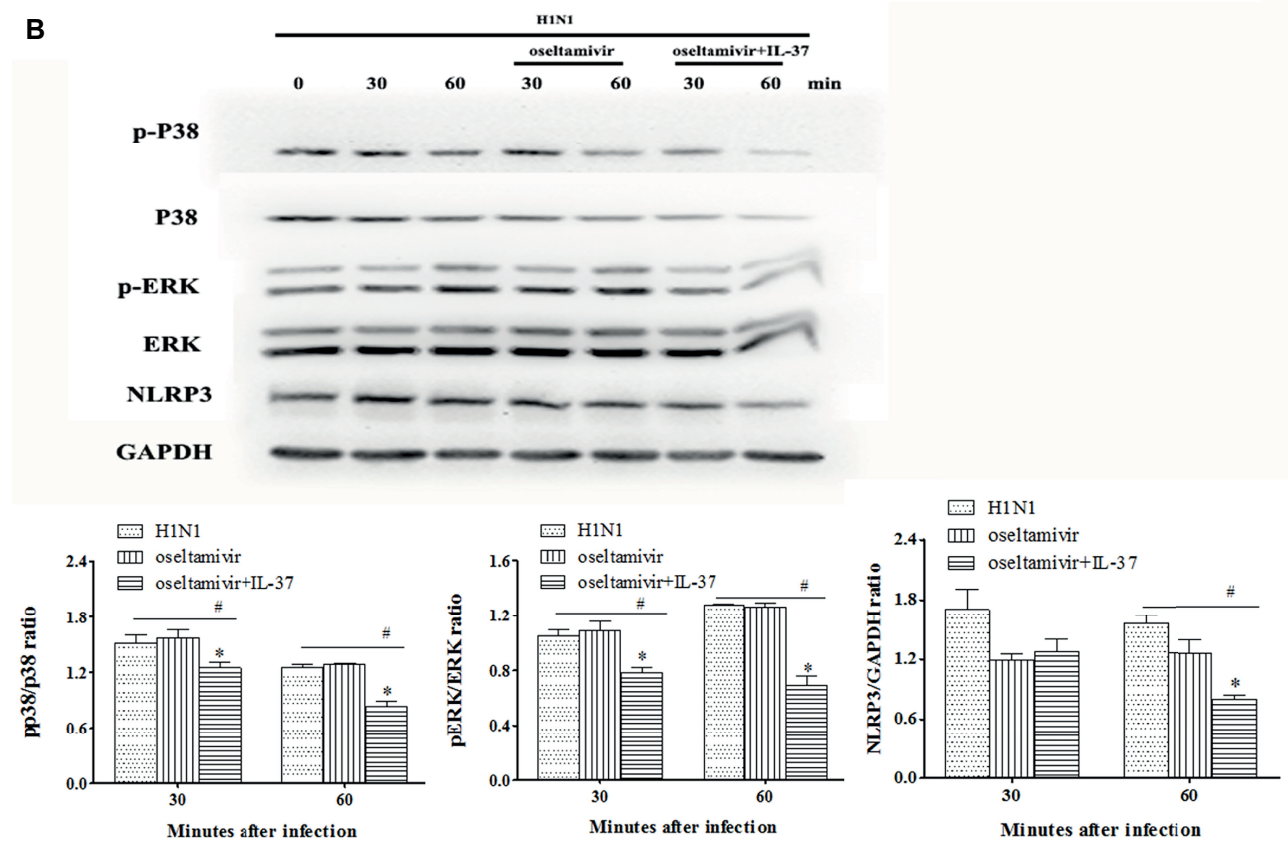

FIGURE 7 | IL-37 inhibited the expression of inflammatory cytokines in MAPK-dependent pathway in vitro. (A) Effects of IL-37 on the mRNA expression of MCP-1, IL-6, MIP-1 $\alpha$, MIP-1 $\beta$, TNF- $\alpha$, etc. in RAW264.7 cells on 12, and $24 \mathrm{~h}$ after infection. (B) Representative photographs of western blot in protein expressions of the phosphorylated and non-phosphorylated forms of ERK1/2, P38 MAPK, NLRP3 and GAPDH in RAW264.7 cells with or without IL-37 treatment during H1N1 infection. The data are representative of three independent experiments. *Significant difference $(p<0.05)$, compared with oseltamivir-inoculated mice. "Significant difference $(p<0.05)$, compared with $\mathrm{H} 1 \mathrm{~N} 1$-infected cells.

In the present study, by using H1N1-infected BALB/c mice, we found that intravenous IL-37 treatment advanced the time to body weight recovery onset, improved the survival rate (Figures 1, 2), and ameliorated the increase in the exudation of inflammatory proteins in the alveoli (Figure 3 ). These results demonstrate that IL-37 treatment can ameliorate viral pneumonia and afford a better protection from A/California/07/2009 (H1N1) infection in the murine model. Furthermore, IL-37 treatment significantly reduced the production of the inflammatory cytokines and chemokines MCP-1, IL-1 $\beta$, MIP-1, IFN- $\gamma$, MIG and RANTES, 
meanwhile the increased mRNA expression of MCP-1, IL- $1 \beta$, IP-10, IL-10, MIG and RANTES in the lungs of the IL-37treatment group was obviously decreased compared with that in the oseltamivir group (Figure 4). Interestingly, most of the cytokines obviously decreased at both the transcriptional and translational levels were macrophage cytokines.

Indeed, IL-37 administration impaired the decrease in the percentage of macrophages in the lungs of H1N1-infected mice (Figure 5), and depleting macrophages reduced the protective effect of IL-37 during influenza virus infection (Figure 6). The anti-inflammatory endogenous ligand annexin A1 has been shown to attenuate pathology upon subsequent influenza A virus infection, and reduction in lung damage severity is associated with an increase in the number of alveolar macrophages (AMs) in the murine model of influenza $\mathrm{A}$ virus infection (Schloer et al., 2019). Numerous literatures have demonstrated that macrophage are critical for host defense in mice during influenza viral infection (He et al., 2017; Wong and Smith, 2017). Our results are consistent with the results of these reports, showing that macrophages may exert immune protective effects in H1N1-infected mice treated with IL-37.

IL-37 can strongly regulate macrophages to restrain the autoimmune response (Ye and Huang, 2015; Toulmin et al., 2017; Wang et al., 2018; Yang et al., 2019). It has been reported that IL-37 can promote macrophage polarization from the pro-inflammatory subtype (M1) to the anti-inflammatory subtype (M2) in atherosclerosis (McCurdy and Baumer, 2017). Moreover, IL-37 induces a phenotypic shift in THP1-derived macrophages toward a CD206 $6^{\text {thigh }}$ and $\mathrm{CD} 86^{\text {+low }}$ macrophage subtype and enhanced the mRNA levels of IL-10, which are characteristic hallmarks of M2 macrophages (Huang et al., 2015). In summary, these results indicate that IL-37 treatment ameliorates the lung damage by polarizing macrophages from an M1 to an M2 phenotype. Further research regarding the mechanisms of the inhibitory effect of IL-37 is needed.

To further confirm that macrophages play an important role in the anti-inflammatory effect of IL-37, RAW264.7 cells were infected with $\mathrm{H} 1 \mathrm{~N} 1$, and treated with oseltamivir in combination with/without IL-37. At the indicated intervals, macrophages were collected, and the expression of cytokine mRNA in the cells was detected. As shown in Figure 7A, compared with that in the oseltamivir group, the mRNA expression of IL-1 $\beta$, IL- 6 , TNF- $\alpha$ and MIP-1 was obviously downregulated in the IL-37 treatment group. These results are consistent with those of pulmonary studies, which further indicates that IL-37 treatment can ameliorate H1N1-induced inflammation by reducing macrophage cytokine production.

The activity of IL-37 has been reported to largely depend upon IL-18Ra and SIGIRR for the extracellular activation of the anti-inflammatory pathway (Lunding et al., 2015; Zeng et al., 2017). Herein, we found that paralleling the augmented macrophages percentage, the IL-18R ${ }^{+}$-macrophages percentage was enhanced markedly in the lungs of IL-37-treated mice (Figure 5), indicating that IL-37 down-regulates the increased production of pro-inflammatory cytokines in an IL-18Ra activation-dependent manner. Then, by using the RAW264.7 cell line, the underlying mechanisms of the IL-37 effect in macrophages were further investigated. Studies have shown that MAPK-related signaling can be inhibited by IL-37 in activated mast cells (Gallenga et al., 2019). In addition, the intraperitoneal injection of IL-37 significantly decreases the expression of NLRP3 in the mouse lung aspergillosis model (Moretti et al., 2014; Jia and Liu, 2018). Indeed, our results showed that the increased phosphorylation of ERK1/2 and p38 MAPK was significantly downregulated in RAW264.7 cells treated with IL-37; furthermore, the ratio of NLRP3 in the IL-37-treated group was decreased in vitro (Figure 7). In contrast, treatment with IL-37 did not inhibit the production of JNK protein in influenza A virus-infected RAW 264.7 cells (data not shown). These results confirm that IL-37 ameliorates influenza pneumonia by attenuating macrophage cytokine production in a MAPK pathway-dependent manner, especially the ERK1/2 and p38 pathways.

In conclusion, these data provide evidence that IL-37 inhibits the pathogenesis of influenza pneumonia by decreasing the production of essential pro-inflammatory cytokines, indicating a new and promising therapeutic approach for excessively activated immune responses in influenza A infection-induced pneumonia. However, the function of IL-37 in other viral infections, especially serious emergent and re-emerged infectious diseases, remains unclear. Further detailed research remains necessary to fully determine the possible functions of IL-37 in viral infections.

\section{DATA AVAILABILITY STATEMENT}

All datasets generated for this study are included in the article/Supplementary Material.

\section{ETHICS STATEMENT}

The animal study was reviewed and approved by The Institute of Animal Use and Care Committee of the Institute of Laboratory Animal Science, Peking Union Medical College.

\section{AUTHOR CONTRIBUTIONS}

$\mathrm{CQ}, \mathrm{LB}$, and FQ conceived, designed, and supervised the experiments. FQ and ML performed most experiments, analyzed the data, and wrote the original draft. $\mathrm{LB}, \mathrm{FQ}$, and $\mathrm{ML}$ analyzed the data. FL, QL, GW, SG, SW, and YX conducted some experiments. FQ and ML edited the manuscript. All authors reviewed and approved the manuscript.

\section{FUNDING}

This work was supported by the National Megaprojects of China for Major Infectious Diseases (2018ZX10301403-004), the Chinese National Major S \& T Project (2017ZX10304402001), the CAMS Innovation Fund for Medical Sciences (2016I2M-1-014, 2016-12 M-006), and the Fundamental Research Funds for the Central Universities (3332018108). 


\section{SUPPLEMENTARY MATERIAL}

The Supplementary Material for this article can be found online at: https://www.frontiersin.org/articles/10.3389/fmicb.2019.02482/ full\#supplementary-material

\section{REFERENCES}

Al-Anazi, M. R., Matou-Nasri, S., Al-Qahtani, A. A., Alghamdi, J., Abdo, A. A., Sanai, F. M., et al. (2019). Association between IL-37 gene polymorphisms and risk of HBV-related liver disease in a Saudi Arabian population. Sci. Rep. 9:7123. doi: 10.1038/s41598-019-42808-4

An, B., Liu, X., Li, G., and Yuan, H. (2017). Interleukin-37 ameliorates coxsackievirus B3-induced viral myocarditis by modulating the Th17/regulatory T cell immune response. J. Cardiovasc. Pharmacol. 69, 305-313. doi: 10.1097/ FJC.0000000000000476

Barbier, L., Ferhat, M., Salame, E., Robin, A., Herbelin, A., Gombert, J. M., et al. (2019). Interleukin-1 family cytokines: keystones in liver inflammatory diseases. Front. Immunol. 10:2014. doi: 10.3389/fimmu.2019.02014

Boraschi, D., Lucchesi, D., Hainzl, S., Leitner, M., Maier, E., Mangelberger, D., et al. (2011). IL-37: a new anti-inflammatory cytokine of the IL-1 family. Eur. Cytokine Netw. 22, 127-147. doi: 10.1684/ecn.2011.0288

Boraschi, D., and Tagliabue, A. (2013). The interleukin-1 receptor family. Semin. Immunol. 25, 394-407. doi: 10.1016/j.smim.2013.10.023

Daoud, A., Laktineh, A., Macrander, C., Mushtaq, A., and Soubani, A. O. (2019). Pulmonary complications of influenza infection: a targeted narrative review. Postgrad. Med. 131, 299-308. doi: 10.1080/00325481.2019.1592400

Davis, C. J., Zielinski, M. R., Dunbrasky, D., Taishi, P., Dinarello, C. A., and Krueger, J. M. (2017). Interleukin 37 expression in mice alters sleep responses to inflammatory agents and influenza virus infection. Neurobiol. Sleep Circadian Rhythms 3, 1-9. doi: 10.1016/j.nbscr.2016.11.005

Dinarello, C. A., and Bufler, P. (2013). Interleukin-37. Semin. Immunol. 25, 466-468. doi: 10.1016/j.smim.2013.10.004

Gallenga, C. E., Pandolfi, F., Caraffa, A., Kritas, S. K., Ronconi, G., Toniato, E., et al. (2019). Interleukin-1 family cytokines and mast cells: activation and inhibition. J. Biol. Regul. Homeost. Agents 33, 1-6. https://www.ncbi.nlm. nih.gov/pubmed/30656901

He, W., Chen, C. J., Mullarkey, C. E., Hamilton, J. R., Wong, C. K., Leon, P. E., et al. (2017). Alveolar macrophages are critical for broadly-reactive antibodymediated protection against influenza A virus in mice. Nat Commun. 8:846. doi: 10.1038/s41467-017-00928-3

Hermann, B., Lehners, N., Brodhun, M., Boden, K., Hochhaus, A., Kochanek, M., et al. (2017). Influenza virus infections in patients with malignancies characteristics and outcome of the season 2014/15. A survey conducted by the infectious diseases working party (AGIHO) of the German Society of Haematology and Medical Oncology (DGHO). Eur. J. Clin. Microbiol. Infect. Dis. 36, 565-573. doi: 10.1007/s10096-016-2833-3

Huang, Z., Gao, C., Chi, X., Hu, Y. W., Zheng, L., Zeng, T., et al. (2015). IL-37 expression is upregulated in patients with tuberculosis and induces macrophages towards an M2-like phenotype. Scand. J. Immunol. 82, 370-379. doi: $10.1111 /$ sji. 12326

Jia, H., and Liu, J. (2018). Reviews of Interleukin-37: functions, receptors, and roles in diseases. Biomed. Res. Int. 2018:3058640. doi: 10.1155/ 2018/3058640

Kumar, S., Hanning, C. R., Brigham-Burke, M. R., Rieman, D. J., Lehr, R., Khandekar, S., et al. (2002). Interleukin-1F7B (IL-1H4/IL-1F7) is processed by caspase- 1 and mature IL-1F7B binds to the IL-18 receptor but does not induce IFN-gamma production. Cytokine 18, 61-71. doi: 10.1006/cyto.2002.0873

Liu, L., Oza, S., Hogan, D., Chu, Y., Perin, J., Zhu, J., et al. (2016). Global, regional, and national causes of under-5 mortality in 2000-15: an updated systematic analysis with implications for the sustainable development goals. Lancet 388, 3027-3035. doi: 10.1016/S0140-6736(16)31593-8

Lunding, L., Webering, S., Vock, C., Schroder, A., Raedler, D., Schaub, B., et al. (2015). IL-37 requires IL-18Ralpha and SIGIRR/IL-1R8 to diminish allergic airway inflammation in mice. Allergy 70, 366-373. doi: 10.1111/ all.12566
SUPPLEMENTARY FIGURE S1 | Percentages of lymphocytes were detected in IL-37-treated mice. (A) The percentages of different lymphocytes that were identified as $\mathrm{CD}^{+} \mathrm{CD}^{+}$or $\mathrm{CD}^{+}{ }^{+} \mathrm{CD} 8^{+}$as well as IL-18R $\alpha^{+}$lymphocytes in the lungs of IL-37 treated mice were determined by flow cytometry on day 6 during $\mathrm{H} 1 \mathrm{~N} 1$ infection. Data are representative of three independent experiments with three mice for each group. *Significant difference $(p<0.05)$, compared with oseltamivir-treated mice.

Lv, J., Xiong, Y., Li, W., Cui, X., Cheng, X., Leng, Q., et al. (2018). IL-37 inhibits IL-4/IL-13-induced CCL11 production and lung eosinophilia in murine allergic asthma. Allergy 73, 1642-1652. doi: 10.1111/all.13395

McCurdy, S., and Baumer, Y. (2017). Macrophage-specific expression of IL-37 in hyperlipidemic mice attenuates. Atherosclerosis 199, 3604-3613. doi: 10.4049/ jimmunol.1601907

Moretti, S., Bozza, S., Oikonomou, V., Renga, G., Casagrande, A., Iannitti, R. G., et al. (2014). IL-37 inhibits inflammasome activation and disease severity in murine aspergillosis. PLoS Pathog. 10:e1004462. doi: 10.1371/journal. ppat.1004462

Morita, M., Kuba, K., Ichikawa, A., Nakayama, M., Katahira, J., Iwamoto, R., et al. (2013). The lipid mediator protectin D1 inhibits influenza virus replication and improves severe influenza. Cell 153, 112-125. doi: 10.1016/j. cell.2013.02.027

Nold, M. F., Nold-Petry, C. A., Zepp, J. A., Palmer, B. E., Bufler, P., and Dinarello, C. A. (2010). IL-37 is a fundamental inhibitor of innate immunity. Nat. Immunol. 11, 1014-1022. doi: 10.1038/ni.1944

Nold-Petry, C. A., Lo, C. Y., Rudloff, I., Elgass, K. D., Li, S., and Gantier, M. P. (2015). IL-37 requires the receptors IL-18Ralpha and IL-1R8 (SIGIRR) to carry out its multifaceted anti-inflammatory program upon innate signal transduction. Nat. Immunol. 16, 354-365. doi: 10.1038/ni.3103

Perez-Padilla, R., De La Rosa-Zamboni, D., Ponce De Leon, S., Hernandez, M., Quinones-Falconi, F., Bautista, E., et al. (2009). Pneumonia and respiratory failure from swine-origin influenza A (H1N1) in Mexico. N. Engl. J. Med. 361, 680-689. doi: 10.1056/NEJMoa0904252

Ramsey, C. D., and Kumar, A. (2013). Influenza and endemic viral pneumonia. Crit. Care Clin. 29, 1069-1086. doi: 10.1016/j.ccc.2013.06.003

Rello, J., Rodriguez, A., Ibanez, P., Socias, L., Cebrian, J., Marques, A., et al. (2009). Intensive care adult patients with severe respiratory failure caused by influenza a $(\mathrm{H} 1 \mathrm{N1}) \mathrm{v}$ in Spain. Crit. Care 13:R148. doi: 10.1186/cc8044

Rice, T. W., Rubinson, L., Uyeki, T. M., Vaughn, F. L., John, B. B., Miller, R. R. 3rd, et al. (2012). Critical illness from 2009 pandemic influenza A virus and bacterial coinfection in the United States. Crit. Care Med. 40, 1487-1498. doi: 10.1097/CCM.0b013e3182416f23

Schloer, S., Hubel, N., Masemann, D., Pajonczyk, D., Brunotte, L., Ehrhardt, C., et al. (2019). The annexin A1/FPR2 signaling axis expands alveolar macrophages, limits viral replication, and attenuates pathogenesis in the murine influenza A virus infection model. FASEB J. 33:fj201901265R. doi: 10.1096/fj.201901265R

Tate, M. D., Pickett, D. L., Van Rooijen, N., Brooks, A. G., and Reading, P. C. (2010). Critical role of airway macrophages in modulating disease severity during influenza virus infection of mice. J. Virol. 84, 7569-7580. doi: 10.1128/ JVI.00291-10

Teng, X., Hu, Z., Wei, X., Wang, Z., Guan, T., Liu, N., et al. (2014). IL-37 ameliorates the inflammatory process in psoriasis by suppressing proinflammatory cytokine production. J. Immunol. 192, 1815-1823. doi: 10.4049/jimmunol.1300047

Theoharides, T. C., Tsilioni, I., and Conti, P. (2019). Mast cells may regulate the anti-inflammatory activity of IL-37. Int. J. Mol. Sci. 20:3701. doi: 10.3390/ ijms 20153701

Toulmin, E., Lee, B. H., and Boisvert, W. A. (2017). Macrophage-specific expression of IL-37 in hyperlipidemic mice attenuates atherosclerosis. J. Immunol. 199, 3604-3613. doi: 10.4049/jimmunol.1601907

Uematsu, T., Iizasa, E., Kobayashi, N., Yoshida, H., and Hara, H. (2015). Loss of CARD9-mediated innate activation attenuates severe influenza pneumonia without compromising host viral immunity. Sci. Rep. 5:17577. doi: 10.1038/ srep 17577

Wang, L., Quan, Y., Yue, Y., Heng, X., and Che, F. (2018). Interleukin-37: a crucial cytokine with multiple roles in disease and potentially clinical therapy. Oncol. Lett. 15, 4711-4719. doi: 10.3892/ol.2018.7982 
Wong, C. K., and Smith, C. A. (2017). Aging impairs alveolar macrophage phagocytosis and increases influenza-induced mortality in mice. J. Immunol. 199, 1060-1068. doi: 10.4049/jimmunol.1700397

Yang, Z., Kang, L., Wang, Y., Xiang, J., Wu, Q., Xu, C., et al. (2019). Role of IL-37 in cardiovascular disease inflammation. Can. J. Cardiol. 35, 923-930. doi: 10.1016/j.cjca.2019.04.007

Ye, L., and Huang, Z. (2015). IL-37 restrains autoimmune diseases. Oncotarget 6, 21775-21776. doi: 10.18632/oncotarget.4887

Zeng, Q., Song, R., Fullerton, D. A., Ao, L., Zhai, Y., Li, S., et al. (2017). Interleukin-37 suppresses the osteogenic responses of human aortic valve interstitial cells in vitro and alleviates valve lesions in mice. Proc. Natl. Acad. Sci. USA 114, 1631-1636. doi: 10.1073/pnas.1619667114

Zhang, S. R., Nold, M. F., Tang, S. C., Bui, C. B., Nold, C. A., Arumugam, T. V., et al. (2019). IL-37 increases in patients after ischemic stroke and protects from inflammatory brain injury, motor impairment and lung infection in mice. Sci. Rep. 9:6922. doi: 10.1038/s41598-019-43364-7

Conflict of Interest: The authors declare that the research was conducted in the absence of any commercial or financial relationships that could be construed as a potential conflict of interest.

Copyright (c) 2019 Qi, Liu, Li, Lv, Wang, Gong, Wang, Xu, Bao and Qin. This is an open-access article distributed under the terms of the Creative Commons Attribution License (CC BY). The use, distribution or reproduction in other forums is permitted, provided the original author(s) and the copyright owner(s) are credited and that the original publication in this journal is cited, in accordance with accepted academic practice. No use, distribution or reproduction is permitted which does not comply with these terms. 